\title{
TRANSMISSION AND REFLECTION OF MILLIMETER WAVES IN $i$-GaAs/ $n$-GaAs PERIODIC STRUCTURES IN VOIGT CONFIGURATION
}

\author{
R. Narkowicz, R. Brazis* and L. Safonova
}

Semiconductor Physics Institute, A. Gostauto 11, 2600 Vilnius, Lithuania

The paper presents the first experimental results on the in-plane propagation of millimeter waves in GaAs ...n-i-n-i-... type periodic layered structures in the Voigt geometry. The data are found to agree with theoretical calculations based on the effective medium approximation, showing the feasibility of contactless non-destructive probing of periodic layered structures.

PACS numbers: 78.66.-w

\section{Introduction}

Layered periodic semiconductor structures continue to attract attention, owing to the possibility of tailoring their bands of interaction with electromagnetic waves so as to fit the requirements of microwave- or optoelectronics [1-8]. The symmetry of periodic structures (PS) comprised of isotropic materials is known to be uniaxial along the axis normal to the layers. Further reduction of symmetry occurs in steady magnetic fields, which leads to gyrotropy of the PS. This opens new possibilities of PS parameter control. Among the various experimental fieldand wave-vector orientations, in-plane propagation in the Voigt geometry is the most attractive, owing to its convenience for experimental realization and data analysis. The wave dispersion in magnetoactive PS composed of technologically advanced materials, such as GaAs, has been considered theoretically in $[5,7]$ and other earlier studies.

In this paper we present the first experimental results on millimeter wave transmission and reflection in PS of ...n-i-n-i-.. type GaAs in the Voigt geometry, involving in-plane propagation. We compare the data with existing theory, with a view of using this approach for characterization of composite materials.

*E-mail: brazis@uj.pfi.lt 


\section{Experiment}

$n$-type GaAs layers grown by the liquid-phase-epitaxy on insulating $i$-type $\mathrm{GaAs}$ substrates were used for the periodic structure sample preparation. The plates were positioned in stack, so as to form a layered $\ldots n-i-n-i-\ldots$ type periodic structure. The $n$-type layer thickness $d_{1}$ in the reported structures was $1 \mu \mathrm{m}$ and $10 \mu \mathrm{m}$, the carrier density $n=10^{16} \mathrm{~cm}^{-3}$ and $2 \times 10^{15} \mathrm{~cm}^{-3}$, and the electron mobility was in the range of 4000 to $6000 \mathrm{~cm}^{2} \mathrm{~V}^{-1} \mathrm{~s}^{-1}$, respectively. The substrate thickness $d_{2}$ was $400 \mu \mathrm{m}$ in all the structures. The PS sample thickness along the wave propagation direction was $5 \mathrm{~mm}$. The samples were positioned between the flanges of standard metallic waveguides, so as to cover completely the waveguide aperture. Lossy dielectric (textolite) sample holders and heavily absorbing diaphragms were used for suppressing any wave leakage. Backward-wave oscillators with output power below $100 \mathrm{~mW}$, and the Schottky-barrier detectors with calibrated directional couplers and attenuators were used, providing the possibility of attenuation measurements down to $-50 \mathrm{~dB}$. A steady magnetic field was applied perpendicular to the direction of wave propagation.

Power transmission and reflection coefficients $T$ and $R$ were measured for two sample orientations. In the first case the uniaxis was oriented perpendicular to the magnetic field. The wave electric field in the PS in this case was oriented perpendicular to the layers. The transmission coefficient (Fig. 1) is found to possess a resonance minimum which turns out to be below the plasma frequency of $n$-type GaAs $\left(f_{\mathrm{p}}=980 \mathrm{GHz}\right.$, and $438.5 \mathrm{GHz}$ for the samples with $n=10^{16} \mathrm{~cm}^{-3}$ and $2 \times 10^{15} \mathrm{~cm}^{-3}$, respectively). Actually the attenuation which equals to $10 \log T$ is
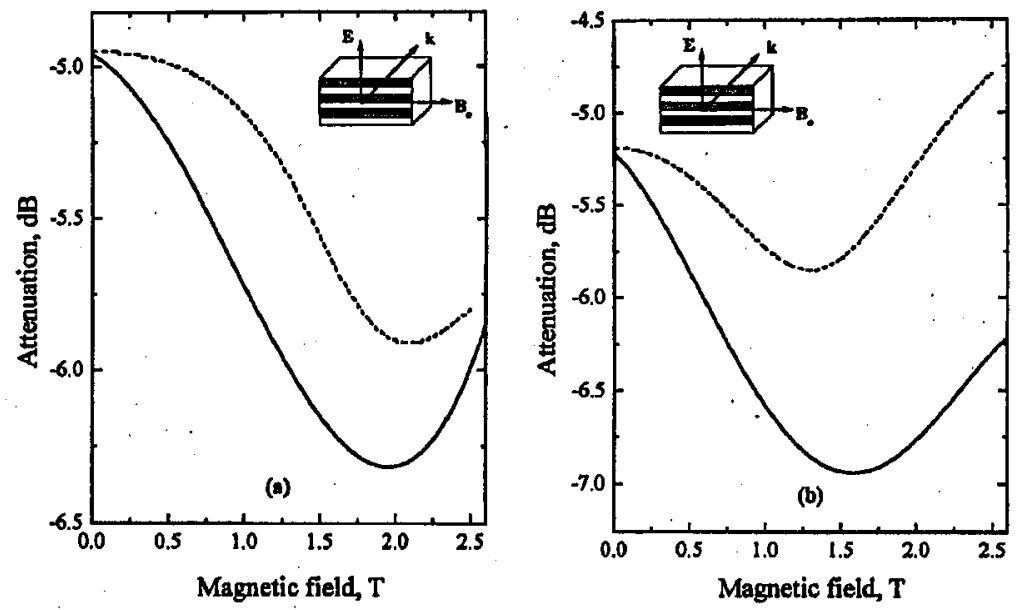

Fig. 1. Transmitted power attenuation as a function of magnetic field for waves polarized normally to the PS layers in the Voigt geometry for the structure with $n=2 \times 10^{15} \mathrm{~cm}^{-3} ; d_{1}=10 \mu \mathrm{m}, d_{2}=400 \mu \mathrm{m}$, solid curves - experiment, dashed - theory; $T=80 \mathrm{~K}$; (a) $f=38 \mathrm{GHz}$, (b) $f=52 \mathrm{GHz}$. PS layers orientation with respect to the wave electric field $\boldsymbol{E}$, the wave vector $k$ and the steady magnetic field $\boldsymbol{B}_{0}$ is shown in the inset. 


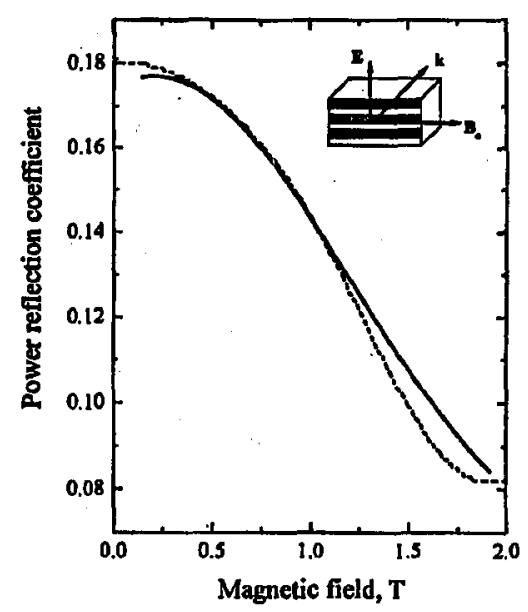

Fig. 2. Power reflection coefficient as a function of magnetic field for the same structure as in Fig. 1, solid curves - experiment, dashed - theory; $T=80 \mathrm{~K}, f=38 \mathrm{GHz}$. PS layers orientation with respect to the wave electric field $E$, the wave vector $k$ and the steady magnetic field $\boldsymbol{B}_{0}$ is shown in the inset.
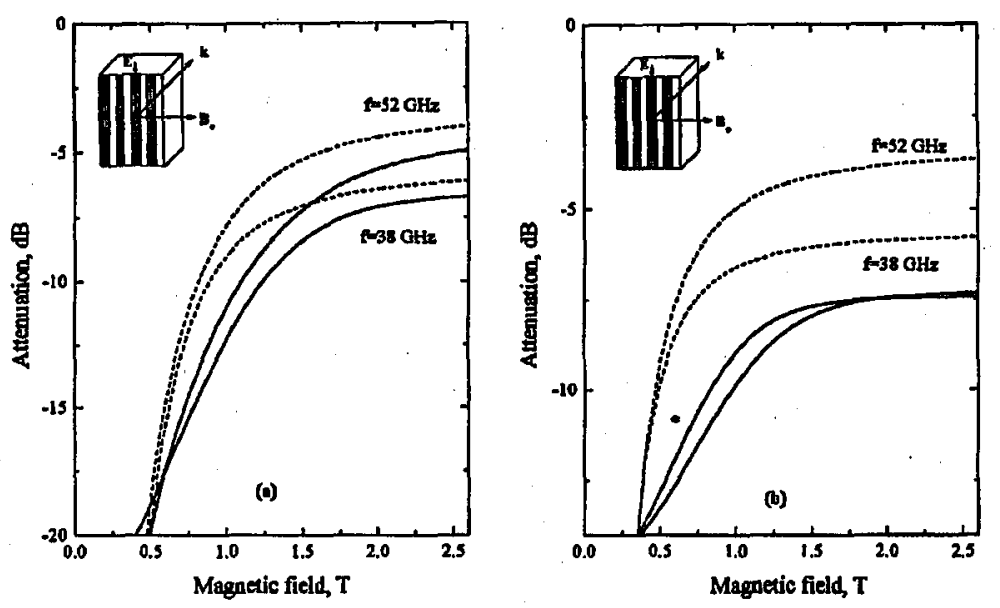

Fig. 3. Transmitted power attenuation as a function of magnetic field for waves polarized parallel to the PS layers in the Voigt geometry for (a) $n=2 \times 10^{15} \mathrm{~cm}^{-3}, d_{1}=$ $10 \mu \mathrm{m}, d_{2}=400 \mu \mathrm{m}$; (b) $n=10^{16} \mathrm{~cm}^{-3}, d_{1}=1 \mu \mathrm{m}, d_{2}=400 \mu \mathrm{m}$, solid curves experiment, dashed - theory. Microwave frequencies are shown next to the curves. PS layers orientation with respect to the wave electric field $\boldsymbol{E}$, the wave vector $k$ and the steady magnetic field $B_{0}$ is shown in the inset.

plotted. The value of magnetic field corresponding to the minimum goes down with increasing frequency (Fig. 1a,b). Note that the resonance is peculiar to periodic structures only. It has no analogy in a continuous $n$-type GaAs (bulk) material 
which is opaque for the millimeter waves in the magnetic fields employed in our experiments. The reflection coefficient in PS is found to decrease when the magnetic field approaches the resonance value (Fig. 2). It means that the periodic structure exhibits a band of heavy absorption related to the resonance.

In the case of the uniaxis orientation along the magnetic field, the layers are parallel to the electric field of the wave, and the wave transmission is suppressed unless the magnetic field is above a threshold value (Fig. 3). The threshold is low: $B_{0}=0.4-0.5 \mathrm{~T}$ for PS with $n=10^{16} \mathrm{~cm}^{-3}$ and $n=2 \times 10^{15} \mathrm{~cm}^{-3}$. The calculated cut-off value of $B_{0}$ in a uniform $n$-type GaAs with the carrier density $n=2 \times 10^{15} \mathrm{~cm}^{-3}$ is $8.5 \mathrm{~T}$ at $52 \mathrm{GHz}$ and $11.7 \mathrm{~T}$ at $38 \mathrm{GHz}$. For the case of $n=10^{16} \mathrm{~cm}^{-3}$ the values are $43 \mathrm{~T}$ and $59 \mathrm{~T}$, respectively, i.e., it is by two orders of magnitude higher than the observed threshold in PS. The threshold- and resonance values of magnetic field are determined both by the carrier density and the layer thickness ratio, as follows from the theory given below.

\section{Theory}

The experimental results are analysed in terms of the eflective medium approximation. The $n$-type GaAs is characterized by the non-zero dielectric tensor components [9]

$$
\begin{aligned}
& \varepsilon_{x x}=\varepsilon_{y y}=\varepsilon_{\mathrm{L}}\left\{1-\frac{\omega_{\mathrm{p}}^{2}(\omega-\mathrm{i} \nu)}{\omega\left[(\omega-\mathrm{i} \nu)^{2}-\omega_{\mathrm{c}}^{2}\right]}\right\}, \\
& \varepsilon_{x y}=-\varepsilon_{y x}=\mathrm{i} \varepsilon_{\mathrm{L}} \frac{\omega_{\mathrm{p}}^{2} \omega_{\mathrm{c}}}{\omega\left[(\omega-\mathrm{i} \nu)^{2}-\omega_{\mathrm{c}}^{2}\right]},
\end{aligned}
$$

where $\omega_{\mathrm{c}}=e B_{0} / m^{*}$ isthe cyclotron frequency, $\omega_{\mathrm{p}}=\left(e^{2} n / m^{*} \varepsilon_{0} \varepsilon_{\mathrm{L}}\right)^{1 / 2}$ is the plasma frequency, $m^{*}$ is the carrier effective mass, $e$ is the elementary charge, $n$ is the free carrier density, $\nu$ is the collision frequency, $\varepsilon_{0}$ is the free-space dielectric constant, and $\varepsilon_{L}$ is the lattice dielectric permittivity determined by phonons and other contributions except the free carriers.

In the case of magnetic field perpendicular to the uniaxis, the effective dielectric tensor of a dielectric-semiconductor PS is given and verified in $[2,3]$. We write it here in the form

$$
\begin{gathered}
\bar{\varepsilon}_{x x}=\frac{\varepsilon_{x x 1} \varepsilon_{x x 2}}{\alpha_{2} \varepsilon_{x x 1}+\alpha_{1} \varepsilon_{x x 2}}, \\
\bar{\varepsilon}_{y x}=-\bar{\varepsilon}_{x y}=\frac{\alpha_{1} \varepsilon_{y x 1} \varepsilon_{x x 2}+\alpha_{2} \varepsilon_{y x 2} \varepsilon_{x x 1}}{\alpha_{2} \varepsilon_{x x 1}+\alpha_{1} \varepsilon_{x x 2}} \\
\bar{\varepsilon}_{y y}=\alpha_{1} \varepsilon_{y y 1}+\alpha_{2} \varepsilon_{y y 2}+\frac{\alpha_{1} \alpha_{2}\left(\varepsilon_{y x 1} \varepsilon_{x y 2}+\varepsilon_{y x 2} \varepsilon_{x y 1}-\varepsilon_{x y 1} \varepsilon_{y x 1}-\varepsilon_{x y 2} \varepsilon_{y x 2}\right)}{\alpha_{2} \varepsilon_{x x 1}+\alpha_{1} \varepsilon_{x x 2}}
\end{gathered}
$$

where $\varepsilon_{x x i}, \varepsilon_{y y i}, \varepsilon_{x y i}, \varepsilon_{y x i}$ are the dielectric tensor components of the layer materials defined by Eqs. (1) and (2), and $\alpha_{1,2}=d_{1,2} /\left(d_{1}+d_{2}\right)$ is the filling factor.

In the case of magnetic field parallel to the uniaxis, the dielectric components are simple averages of the same components of the constituent materials

$$
\bar{\varepsilon}_{x x}=\bar{\varepsilon}_{y y}=\alpha_{1} \varepsilon_{x x 1} d_{1}+\alpha_{2} \varepsilon_{x x 2},
$$




$$
\bar{\varepsilon}_{x y}=-\bar{\varepsilon}_{y x}=\alpha_{1} \varepsilon_{x y 1} d_{1}+\alpha_{2} \varepsilon_{x y 2} .
$$

The Voigt dielectric constant in both cases is standard [9],

$$
\varepsilon_{\nu}=\frac{\bar{\varepsilon}_{x x} \bar{\varepsilon}_{y y}-\bar{\varepsilon}_{x y} \bar{\varepsilon}_{y x}}{\bar{\varepsilon}_{y y}} .
$$

Using this expression together with the Fresnel formulas for normal incidence of the waves on a slab, the wave transmission and reflection coefficients are calculated, taking the parameter values for $\varepsilon_{\mathrm{L}}=12.9, m^{*}=0.065 m_{\mathrm{e}}$, and $\nu=8 \times 10^{11} \mathrm{~s}^{-1}$ for all the samples. The theoretical results are seen to reproduce the main experimental features (Figs. 1-3). Further improvement of the agreement between theory and experiment can be achieved by refining the fitting procedure, by including the buffer layers and by taking into account deviations from the assumed step-like change of the carrier density. An interplay between different scattering mechanisms controlled by the lattice temperature, the compensation level of the GaAs, the actual electric and magnetic fields influencing the carrier energy, etc., should be included in a rigorous analysis. Their considerations is, however, beyond the scope of this paper, which focuses on the main properties of the magnetoactive PS.

\section{Conclusions}

The effective medium model is applicable to a PS where the wavelength or, more rigorously, the constant of wave amplitude decay across the layers, is large compared to the spatial period of the structure. In our experiments on the large-period PS, this requirement has been fulfilled rather approximately. The reasonable agreement between theory and the present experiments enables one to apply the modeling for studies of small-period structures obtainable in modern semiconductor technologies.

One of the most striking properties of the PS is their transparency for the waves polarized parallel to the conducting layers arising in the presence of magnetic fields, which occurs at fields two orders of magnitude lower than in the case for an uniform (i.e. bulk) semiconductor. This feature, observed in experiments on the large-period structures, is applicable to the small-period PS with two-dimensional conduction layers as well.

The resonance observed for the waves polarized normally to the layers provides an additional convenient tool for the studies of such modulated structures.

\section{Acknowledgements}

One of us (R.N.) expresses his gratitude for the Józef Mianowski Fund and Foundation for Polish Science for partial support of this work.

\section{References}

[1] P. Yeh, A. Yariv, Chi-Shain Hong, J. Opt. Soc. Am. 67, 423 (1977); A. Yariv, P. Yeh, J. Opt. Soc. Am. 67, 438 (1977).

[2] R. Brazis, in: Semiconductor Multilayer Structures and Superlattices, Eds. A.M. Belyantsev, Yu.A. Romanov, Applied Physics Institute, Gorky 1984, p. 174. 
[3] R. Brazis, L. Safonova, Pis'ma Zh. Eksp. Teor. Fiz. 10, 1325 (1984); Int. J. Infrared Millim. Waves 8, 449 (1987).

[4] V.M. Agranovich, Solid State Commun. 78, 747 (1991).

[5] B.L. Johnson, R.E. Camley, Phys. Rev. B 43, 6554 (1991).

[6] M.S. Kushwaha, B. Djafari Rouhani, Phys. Rev. B 43, 9021 (1991).

[7] R. Brazis, V.E. Lyubchenko, L. Safonova, Radiotekh. Elektron. 38, 1118 (1993).

[8] T. Dumelov, D.R. Tilley, J. Opt. Soc. Am. A 10, 633 (1993).

[9] E.D. Palik, J.K. Furdyna, Rep. Prog. Phys. 33, 1193 (1970). 